\title{
Mechanics of Bundled Semiflexible Polymer Networks
}

\author{
O. Lieleg, ${ }^{1}$ M. M. A. E. Claessens, ${ }^{1}$ C. Heussinger, ${ }^{2}$ E. Frey, ${ }^{2}$ and A. R. Bausch ${ }^{1}$ \\ ${ }^{1}$ Lehrstuhl für Biophysik E22, Technische Universität München, James-Franck-Straße 1, 85748 Garching, Germany \\ ${ }^{2}$ Arnold Sommerfeld Center (ASC) for Theoretical Physics and Center for NanoScience (CeNS), \\ Ludwig-Maximilians-Universität München, Theresienstraße 37, D-80333 München, Germany
}

(Received 27 November 2006; published 22 August 2007)

\begin{abstract}
While actin bundles are used by living cells for structural fortification, the microscopic origin of the elasticity of bundled networks is not understood. Here, we show that above a critical concentration of the actin binding protein fascin, a solution of actin filaments organizes into a pure network of bundles. While the elasticity of weakly cross-linked networks is dominated by the affine deformation of tubes, the network of bundles can be fully understood in terms of nonaffine bending undulations.
\end{abstract}

PACS numbers: 87.15.La

The mechanical properties and dynamic organization of the cytoskeleton determine the morphology and mechanical response of eukaryotic cells. To ensure adaptability of both organization and mechanics, cells exploit the dynamic interplay between semiflexible polymers such as microtubules and actin filaments using a multitude of associated binding proteins. In particular, the local elastic properties are regulated by the activation of auxiliary proteins which, e.g., cross-link and/or bundle the filamentous networks into complex scaffolds. Given the importance of the actin cytoskeleton for force generation and transduction there is much interest in understanding the mechanical properties of different network structures and the physical origin of the transitions between them. This is best studied in in vitro model systems [1]. In the absence of cross-links actin solutions are successfully described by the spatial confinement of thermal bending undulations upon affine tube deformation [2]. Cross-linked semiflexible polymer networks, on the other hand, are in general dominated by an interplay between polymer stretching and bending modes, the precise form of which, as well as the degree of nonaffinity, strongly depends on the network microstructure [3]. So far the mechanical response of highly cross-linked actin networks, also in the presence of bundles and composite phases, has mainly been described assuming purely affine entropic stretching deformations [4-7]. However, an applied tension can stretch a thermally undulating polymer only as far as there is excess contour length available. As the maximal amount of stored length is inversely proportional to the persistence length, entropic stretching is suppressed in networks of stiff polymer bundles, where the persistence length grows with bundle size $[8,9]$. Moreover, the highly nonlinear nature of the force-extension relation of semiflexible polymers implies that linear elasticity is applicable as long as only a fraction of the total excess length is pulled out. As an alternative the recently introduced concept of the "floppy modes" may be better suited to describe the polymer elasticity in situations where entropic effects are suppressed [10]. These floppy modes constitute bending excitations which, unlike the affine stretching deformations, retain a highly nonaffine character.

In this Letter we show that above a critical concentration of the actin binding protein (ABP) fascin a solution of actin filaments organizes into a homogeneous network whose building blocks are bundles only. At low cross-linker concentration, the network response is dominated by the affine deformation of reptation tubes and the ensuing changes in confinement free energy [11]. The observed mechanical and structural transition between both phases can be described by a simple relation between the ABP concentration and the entanglement length. It is proposed to rationalize the scaling of the elastic modulus in the bundled regime in terms of the floppy mode picture. A model based on affine stretching deformations only fits the data if additional assumptions about the bundle structure are made.

G-actin is obtained from rabbit skeletal muscle and stored in lyophilized form at $-21^{\circ} \mathrm{C}$ [12]. For measurements the lyophilized actin is dissolved in deionized water and dialyzed against G-buffer ( $2 \mathrm{~m} M$ Tris, $0.2 \mathrm{~m} M$ adenosine triphosphate (ATP), $0.2 \mathrm{mM} \mathrm{CaCl} 2,0.2 \mathrm{~m} M$ dithiothreitol (DTT), and $0.005 \% \mathrm{NaN}_{3}, p \mathrm{H} 8$ ) at $4{ }^{\circ} \mathrm{C}$. The Gactin solutions are kept at $4{ }^{\circ} \mathrm{C}$ and used within 7 days of preparation. The average length of the actin filaments is controlled to $21 \mu \mathrm{m}$ using gelsolin which is prepared from bovine plasma serum following [13]. Recombinant human fascin $(55 \mathrm{kD})$ was prepared by a modification of the method of [14] as described by [15]. In the experiments the molar ratio $R$ between fascin and actin, $R=c_{f} / c_{a}$, is varied over almost three decades.

To resolve the structure and mechanical properties of actin-fascin networks actin is polymerized in F-buffer (2 $\mathrm{m} M$ Tris, $2 \mathrm{~m} M \mathrm{MgCl}_{2}, 0.2 \mathrm{~m} M \mathrm{CaCl}_{2}, 0.2 \mathrm{~m} M$ DTT, $100 \mathrm{~m} M \mathrm{KCl}$, and $0.5 \mathrm{~m} M$ ATP, $p \mathrm{H}$ 7.5). For fluorescence microscopy filaments are stabilized with tetramethyl rhodamine iso-thiocyanate (TRITC)-phalloidin; either labeled reporter filaments (1 per 400) or continuous labeling is used at distinct amounts of fascin. To avoid photobleaching $0.6 \mu M$ glucose oxidase, $0.03 \mu M$ catalase, and $0.01 M$ glucose are added. The samples for trans- 
mission electron microscopy (Philips EM 400T) are adsorbed to glow-discharged carbon-coated formvar films on copper grids. The samples are washed in a drop of distilled water and negatively stained with $0.8 \%$ uranyl acetate; excess liquid is drained with filter paper. The viscoelastic response of actin-fascin networks is determined by measuring the frequency-dependent viscoelastic moduli $G^{\prime}(\omega)$ and $G^{\prime \prime}(\omega)$ with a stress-controlled rheometer (Physica MCR 301, Anton Paar, Graz, Austria) within a frequency range of three decades. Approximately $520 \mu \mathrm{l}$ sample volume are loaded within $1 \mathrm{~min}$ into the rheometer using a $50 \mathrm{~mm}$ plate-plate geometry with $160 \mu \mathrm{m}$ plate separation. To ensure linear response small torques are applied. Actin polymerization is carried out in situ, measurements are taken $60 \mathrm{~min}$ after the polymerization was initiated.

Fluorescence images show that in the presence of high concentrations of fascin, actin filaments organize into a network of bundles [Fig. 1(a)] while below a critical value $R^{*} \approx 0.01$ no bundles can be observed. Both fluorescence and transmission electron microscopy do not show any signs of composite phases or microdomains as observed in the presence of other ABPs $[6,16,17]$. Moreover, the existence of a purely bundled phase is demonstrated by a cosedimentation assay (see supplementary material [18]). The bundles formed are very long $(>100 \mu \mathrm{m})$ and straight, which is consistent with the measured bending rigidity $\kappa$ [8]. TEM micrographs reveal that above $R^{*}$ the actin-fascin bundle thickness $D$ and therefore the number of actin filaments per bundle $N$ increases weakly with $R$
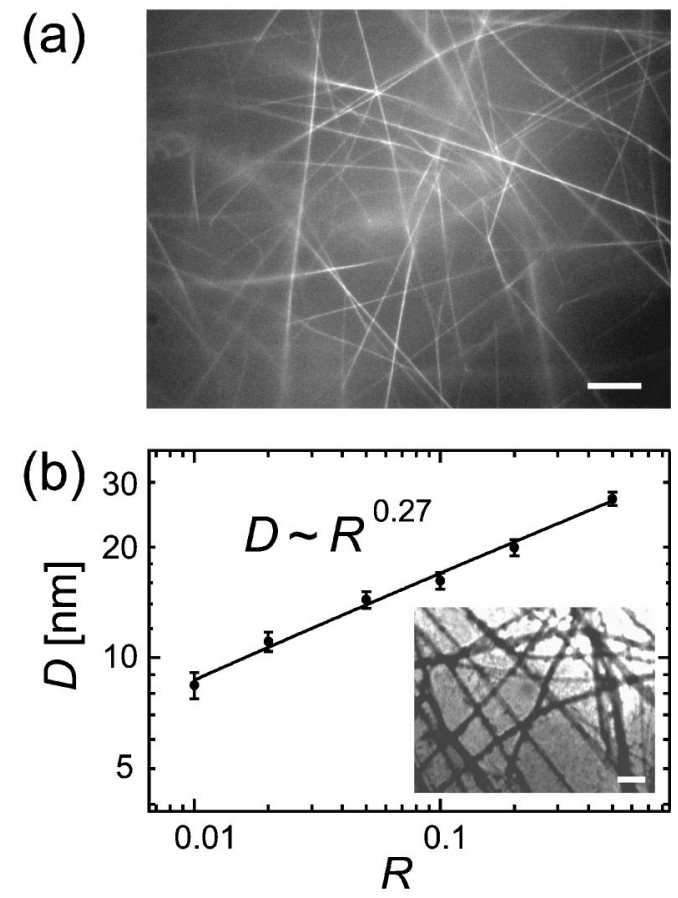

FIG. 1. (a) Fluorescence micrograph of an actin-fascin network $(0.1 \mathrm{mg} / \mathrm{ml}$ actin): for high fascin concentrations a purely bundled network is formed (scale bar is $10 \mu \mathrm{m}$ ). (b) From TEM pictures (inset, scale bar is $0.2 \mu \mathrm{m}$ ) a scaling relation for the average bundle diameter $D$ is obtained.
[Fig. 1(b)]. The bundle thicknesses are extracted from the TEM micrographs by fitting a Gaussian to the intensity profiles, obtaining a scaling of $D \sim N^{1 / 2} \sim R^{x}$ with $x=$ 0.27 . Concomitant with the structural changes the viscoelastic properties of the network alter: with increasing $R$ both the storage modulus $G^{\prime}(\omega)$ and the loss modulus $G^{\prime \prime}(\omega)$ increase over the whole frequency range probed. The storage modulus $G^{\prime}(\omega)$ exhibits a plateau at low frequencies, while the loss modulus $G^{\prime \prime}(\omega)$ reveals a well-defined minimum which shifts to higher frequencies with increasing $R$. The plateau modulus $G^{\prime}(10 \mathrm{mHz})$ plotted against $R$ shows two distinct regimes in the elastic response. At low $R, G_{0}$ is only slightly dependent on $R$, $G_{0} \sim R^{0.1 \pm 0.1}$, while above a critical value $R^{* *}, G_{0}$ increases with $G_{0} \sim R^{1.5 \pm 0.2}$ (Fig. 2). This exponent fits the data for both actin concentrations probed $\left(c_{a}=\right.$ $0.2 \mathrm{mg} / \mathrm{ml}$ and $c_{a}=0.4 \mathrm{mg} / \mathrm{ml}$ ). The transition point $R^{* *}$ agrees well with the structural transition at $R^{*}$ observed in microscopy. Below $R^{*}=R^{* * *}$ the plateau modulus scales with the actin concentration as $G_{0} \sim c_{a}^{1.3}$ suggesting that entanglements dominate the elastic response [2]. Above $R^{*}$ a different scaling regime occurs with $G_{0} \sim c_{a}^{2.4}$.

With the observed scaling behavior $G_{0}\left(R, c_{a}\right)$ the plateau modulus is parametrized in both regimes, before and after the structural transition. At the crossover concentration $R=R^{*}$ these two parametrizations have to be equal. This uniquely determines the scaling of $R^{*}$ with the actin concentration, $R^{*} \sim c_{a}^{-0.79}$, which results in the constraint $c_{f} c_{a}^{-0.21} \sim 1$. This can be approximated to $c_{f} l_{e}^{1 / 2} \sim 1$ using the entanglement length $l_{e} \sim c_{a}^{-2 / 5}$. This surprisingly simple criterion for the bundling transition defies an obvious explanation and a detailed theoretical model is still lacking. It would need to account for the subtle interplay between confinement free energy of polymers in both the

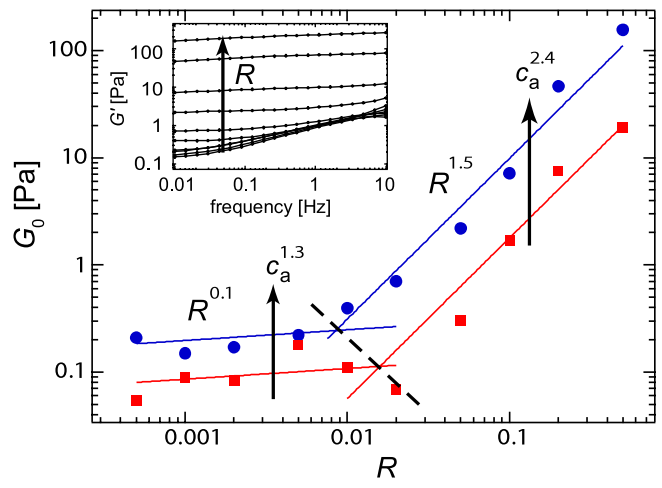

FIG. 2 (color online). Plateau modulus $G_{0}$ as a function of the molar ratio $R$ of fascin with respect to actin for two different concentrations of actin: $0.4 \mathrm{mg} / \mathrm{ml}$ (circles) and $0.2 \mathrm{mg} / \mathrm{ml}$ (squares). The dependence of $G_{0}$ on $c_{a}$ is obtained by scaling the fits for the $0.2 \mathrm{mg} / \mathrm{ml}$ actin data upon the $0.4 \mathrm{mg} / \mathrm{ml}$ data points. The dashed line shows the boundary separating the two scaling regimes. The original frequency spectra for $0.4 \mathrm{mg} / \mathrm{ml}$ actin at different cross-linker concentrations $(R=0,0.001$, $0.002,0.005,0.01,0.02,0.05,0.1,0.2,0.5)$ are depicted in the inset. 
bundle and the network as well as the binding enthalpy of the cross-linking proteins.

The mechanical properties inside the bundled regime may, on the other hand, be understood in terms of the nonaffine floppy mode model [10], where network elasticity is attributed to bending modes of wavelength comparable to the distance between cross-links $l_{c}$ and with stiffness $k_{\perp} \sim \kappa / l_{c}^{3}$. In this picture typical deformations of the network do not follow the macroscopic strain affinely but scale as $\delta_{\text {na }} \sim \gamma L_{B}$, where $L_{B}$ is a constant length over which an individual bundle within the network can be assumed to be straight. From our fluorescence and TEM pictures, we would expect this length to be comparable to the bundle length. As a consequence the linear elastic modulus reads

$$
G_{0} \sim \nu k_{\perp} \delta_{\mathrm{na}}^{2}
$$

with the polymer density $\nu \sim 1 / \xi^{2} l_{c}$. This model can be tested by relating the structural parameters of the network, mesh size $\xi$ and $l_{c}$, and the bending elasticity $\kappa$ of the bundle segment to the concentration of actin and fascin monomers $\left(c_{a}, c_{f}\right)$.

The structural information obtained by TEM and fluorescence microscopy justifies the assumption that the bundles form an isotropic network similar to an entangled structure of single filaments. With increasing $R$, filaments and smaller bundles reorganize to form larger bundles that are spaced farther apart. The mesh size $\xi$ of this selfsimilar structure therefore depends on $R$ as $\xi \sim \xi_{0} N^{1 / 2}$, where $\xi_{0} \sim c_{a}^{-1 / 2}$ is the mesh size of the filamentous network. Cross-linking will typically occur on the scale of the entanglement length $l_{e}$, which plays the role of a distance between bundle-bundle intersections (entanglement points). Since on average only a fraction of those will be occupied we can assume that distances between cross-links along the same bundle are given by $l_{c} \sim R^{-y} l_{e}[6,7]$. Doubling the cross-linker concentration $R$ should halve the distance between them, suggesting an exponent $y \approx 1$.

For a description of the elastic properties of the bundles it is necessary to realize that fascin only leads to loosely coupled bundles, where bending is dominated by the shear stiffness of the cross-linking proteins [8,9]. The key quantity in this context is the bundle coupling parameter $\alpha\left(l_{c}\right)=\left(l_{c} / b\right)^{2}$, where the length scale $b \sim \delta^{1 / 2}$ encodes the properties of the ABPs inside the bundle, in particular via the average distance $\delta$ between cross-links. In general, $\delta$ will depend on the concentrations $c_{f}$ and $c_{a}$; however, the precise relationship is not known. From fluorescence images the mesh size of the bundled network at $R=0.5$ can be approximated which allows one to calculate $l_{e}$ and thus $l_{c}$. From this one can estimate the coupling parameter to be $\alpha>1$ for the whole bundle regime, implying that the effective bundle bending stiffness $\kappa$ acquires a wavelength dependence [9], leading to $\kappa(\lambda) \sim N \kappa_{f} \alpha(\lambda)$, where $\lambda$ is the wavelength of the deformation mode. This stands in marked contrast to what is known for single filaments or scruin-bound bundles where a fully coupled bending re- gime, $\kappa \sim N^{2} \kappa_{f}$, has been assumed [19]. The wavelength dependence of the bundle stiffness has far reaching consequences for the static as well as dynamic properties of semiflexible polymer bundles [20]. In particular, it implies that the entanglement length $l_{e}$ has to be reevaluated. As it derives from the suppression of long wavelength fluctuations by confining a polymer into a tube [21], it is highly sensitive to a wavelength dependent $\kappa(\lambda)$. This results in $l_{e}^{3} \sim\left(N l_{p}\right) \xi^{4} / b^{2}$, which is different from the usual expression $l_{e}^{5} \sim l_{p} \xi^{4}$ valid for single filaments, where in both cases $l_{p}=17 \mu \mathrm{m}$ [22] denotes the persistence length of a single actin filament. Combining the above results and setting the deformation mode length $\lambda$ equal to $l_{c}$, one finally arrives at the following prediction

$$
G_{0} \sim R^{z} c_{a}^{w} \delta^{-1 / 3},
$$

where the exponents are given by $z=2 y-4 x$ and $w=$ $7 / 3$. Thus the scaling exponent of the plateau modulus can be related to parameters describing the microstructure such as the scaling of the mesh size as well as the dependence of the bundle thickness and elasticity on $R$. From our measurements of $x=0.27$ and $z=1.5$, and by assuming $\delta$ to be a constant, a value of $y=1.29$ is obtained, which is in reasonable agreement with the expected $y \approx 1$. This result is largely insensitive to the assumption of constant $\delta$, since by assuming $\delta$ to change according to simple Langmuir kinetics an exponent $y \approx 1.21$ is obtained.

To further characterize the elastic properties in the bundled regime, the nonlinear elasticity of the network is investigated. For samples with $R>R^{*}$ a constant shear rate is applied and the resulting stress is reported. From the smoothed $\sigma(\gamma)$ relation the numerical derivative yielding the differential modulus $K=\partial \sigma / \partial \gamma$ is calculated (Fig. 3). For small strains of $\gamma=1 \%-10 \%$ linear behavior is observed, where the differential modulus follows $K \sim R^{1.5}$ in agreement with our oscillatory measurements. A nonlinear response is observed above $\gamma_{c}$, which is determined as the strain at which $K$ deviates by $5 \%$ from its value in the

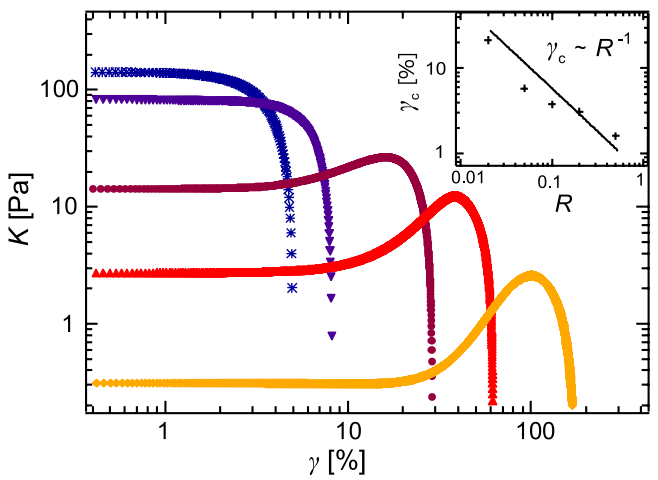

FIG. 3 (color online). Differential modulus $K=d \sigma / d \gamma$ plotted versus deformation $\gamma$ for fascin networks in the bundle phase ( $c_{a}=0.4 \mathrm{mg} / \mathrm{ml}$ and increasing $R$ : diamonds $R=0.02$; upright triangles: $R=0.05$; circles: $R=0.1$; downright triangles: $R=$ 0.2 ; stars: $R=0.5$ ). The inset shows the critical strain $\gamma_{c}$ in dependence on $R$. 
linear regime. Up to $R=0.1$ a strain hardening occurs while for very high values of $R$ the linear regime is directly followed by strain weakening. The disappearance of the strain hardening at high concentrations of fascin might be the result of the rupturing of fascin-actin bonds - very similar to what was reported for rigor heavy meromyosin (HMM) networks [7]. The floppy mode description also has implications on the onset of the nonlinear behavior. As has been argued in [10] large strains necessarily lead to stretching even if the deformations were only of bending character. The bundle stretching $\Delta$ is related to the transverse displacement $\delta_{\text {na }}$ by simple geometric considerations as $l_{c}^{2}+\delta_{\mathrm{na}}^{2}=\left(l_{c}+\Delta\right)^{2}$. The floppy mode description thus applies as long as this stretching is small compared to the available thermal excess length $\Delta \Lambda \sim l_{c} b / N l_{p}$ [20]. This defines a critical strain $\gamma_{c} \sim l_{c}(b / N)^{1 / 2} \sim R^{-y+x} b^{-1 / 6} \sim$ $R^{-1.0} \delta^{-1 / 12}$ for the onset of nonlinear effects. The scaling with $R$ is in excellent agreement with our measurements (see inset of Fig. 3). The weak dependence on the crosslinker spacing $\gamma_{c} \sim \delta^{-1 / 12}$ also implies that this result is insensitive towards any putative dependence on the fascin concentration via $\delta=\delta\left(c_{f}\right)$.

On the other hand, if one were to apply an affine stretching model, a different picture emerges, where $\delta\left(c_{f}\right)$ has to be tuned to obtain a reasonable data fit. In such a model one would assume the modulus to be given by $G_{\text {aff }} \sim \nu k_{\|} \delta_{\text {aff }}$, where $k_{\|} \sim \alpha^{3 / 2}\left(N \kappa_{f}\right)^{2} / l_{c}^{4}$ is the stretching stiffness of the bundle [20]. The deformations are assumed to be affine, implying $\delta_{\text {aff }} \sim \gamma l_{c}$. The modulus thus reads as $G_{\text {aff }} \sim$ $R^{2 x} c_{a} \delta^{-3 / 2}$ [18] while the critical strain $\gamma_{c} \sim \delta^{1 / 2} R^{-2 x}$ is obtained by equating $\Delta \Lambda$ with the affine deformation $\delta_{\text {aff }} \sim \gamma l_{c}$. This model can only fit the data by assuming $\delta \sim c_{f}^{-\beta}$ with an exponent in the range of $\beta=0.6-0.9$, such that for $\beta=0.6$ the $R$ dependence of the modulus and for $\beta=0.9$ the $c_{a}$ dependence of the modulus is reproduced. To finally decide whether or not the application of an affine stretching model is equally successful as the floppy mode approach, $\delta\left(c_{f}\right)$ would have to be determined by scattering experiments.

In summary, on the basis of a combined microscopy and rheology study we have shown that the actin binding protein fascin mediates a transition from an entangled polymer solution to a homogeneously cross-linked bundle phase. These phases differ both in structure and mechanical properties. The location of the transition is given by a simple relation between the ABP concentration and the entanglement length. Moreover, this transition point seems to be more general since it also occurs at similar ABP concentrations in other systems such as isotropically crosslinked networks or even composite networks $[4,7,17]$. The transition is a consequence of the interplay between the chemical kinetics of the binding proteins, the bending rigidity of the polymers, and the entropic forces between those components. How the concerted action of those driving forces leads to such a structural transition is an interesting theoretical problem. The elasticity in the bundled phase is well explained in terms of a recently developed floppy mode picture [10]. We have argued that in the absence of a significant amount of stored length in the bundles, nonaffine bending is the dominant low energy excitation. It explains both the linear elasticity and the onset of nonlinear behavior. This model has to be seen as an alternative to affine models where the elastic response is due to pulling out stored length fluctuations. While the elasticity of isotropic networks may be predominantly determined by such an entropic stretching of single filaments between the cross-linking points, we suppose that the elastic response of composite phases may instead be dominated by nonaffine deformations of bundles as described by floppy modes. The detailed understanding of the presented purely bundled network, composed of shear dominated bundles, provides a benchmark for addressing the further challenge to describe the mechanics of networks, which are dominated by structural heterogeneities.

We thank M. Rusp for the actin preparation. This work was supported by Deutsche Forschungsgemeinschaft through the DFG-Cluster of Excellence Nanosystems Initiative Munich (NIM) and No. SFB 413. Oliver Lieleg was supported by CompInt in the framework of the ENB Bayern.

[1] A. R. Bausch and K. Kroy, Nature Phys. 2, 231 (2006).

[2] B. Hinner et al., Phys. Rev. Lett. 81, 2614 (1998).

[3] C. Heussinger and E. Frey, Phys. Rev. Lett. 96, 017802 (2006).

[4] M. L. Gardel et al., Science 304, 1301 (2004).

[5] F. C. MacKintosh et al., Phys. Rev. Lett. 75, 4425 (1995).

[6] J.H. Shin et al., Proc. Natl. Acad. Sci. U.S.A. 101, 9636 (2004).

[7] R. Tharmann et al., Phys. Rev. Lett. 98, 088103 (2007).

[8] M. M. A. E. Claessens et al., Nat. Mater. 5, 748 (2006).

[9] M. Bathe et al., arXiv:q-bio/0607040.

[10] C. Heussinger and E. Frey, Phys. Rev. Lett. 97, 105501 (2006).

[11] E. Frey, in Advances in Solid State Physics, edited by B. Kramer (Springer, Berlin, 2001), Vol. 41.

[12] J. A. Spudich and S. Watt, J. Biol. Chem. 246, 4866 (1971).

[13] H. Kurokawa et al., Biochem. Biophys. Res. Commun. 168, 451 (1990).

[14] S. Ono et al., J. Biol. Chem. 272, 2527 (1997).

[15] D. Vignjevic et al., J. Cell Biol. 160, 951 (2003).

[16] M. Tempel et al., Phys. Rev. E 54, 1802 (1996).

[17] B. Wagner et al., Proc. Natl. Acad. Sci. U.S.A. 103, 13974 (2006).

[18] See EPAPS Document No. E-PRLTAO-99-069734 for supplementary information. For more information on EPAPS, see http://www.aip.org/pubservs/epaps.html.

[19] J. H. Shin et al., J. Mol. Biol. 337, 255 (2004).

[20] C. Heussinger et al., Phys. Rev. Lett. 99, 048101 (2007).

[21] T. Odijk, Macromolecules 16, 1340 (1983).

[22] L. LeGoff et al., Phys. Rev. Lett. 89, 258101 (2002). 